\title{
Role of MRI in the diagnosis and prognosis of ventricular arrhythmias
}

\author{
Nakul C Sharma*, lan Paterson \\ From 2011 SCMR/Euro CMR Joint Scientific Sessions \\ Nice, France. 3-6 February 2011
}

\section{Background}

Many patients with malignant cardiac arrhythmias' require thorough investigations to determine the etiology. Conventional tests (ECG and echocardiography) often cannot detect a structural basis for the arrhythmia and physicians therefore lack guidance as to the most appropriate treatment.

Previous studies have shown MRI is a more sensitive and specific modality to better elucidate cardiac abnormalities and provide insight to the underlying etiology and better predict clinical outcomes.

\section{Hypothesis}

Compared to conventional testing, Cardiac MRI has incremental benefit in the diagnosis and treatment of patients with ventricular tachycardia and in the assessment/treatment of patients with suspected ARVD.

\section{Methods}

We performed a retrospective review of patients who have undergone CMR as part of their treatment/workup for ventricular arrhythmias and/or ARVD at the University of Alberta Hospital over the last 4 years. CMR results were correlated to patient outcomes: death, cardiac death, hospital admissions, ICD implantation, and electrophysiologic ablation

\section{Results}

A total of 211 patients were enrolled; however complete clinical data on only 142 patients was obtained. The baseline age/sex was 46 years \& $59 \%$ male. The average BSA was $1.86 \mathrm{~m}^{\wedge} 2$. Known CAD was documented in $9.8 \%$.

The presenting symptom(s) to medical attention are as follows: Syncope $36.6 \%$, Palpitation $36.6 \%$, Arrest 9.2\%, None $17.6 \%$. The arrhythmia's documented upon patient

Table 1 Average Ejection fraction based on Imaging Modality

\begin{tabular}{llll}
\hline & Echo & CMR & Atypical Gadolinium uptake \\
\hline LVEF\% & 52.82 & $60.36 \%$ & $58.2 \%$ \\
\hline RVEF\% & Normal & $55.2 \%$ & $53 \%$
\end{tabular}

review were Atrial Tachycardia 2.8\%, 10.5\%, NSVT $28.8 \%$, VT $31.7 \%$, and VF 5.6\%, None 20.6\%)

Based on the clinical presentation $55 \%$ of patients were admitted to hospital, 37\% went on to have an EPS procedure. There were 12 cardiac arrests at presentation that received an ICD. A further $6 \%$ who did not arrest also received an ICD

The corresponding Ejection fractions can be seen in Table 1.

In terms of ARVD, a diagnosis was made in $2.1 \%$ of the patients via CMR while $8.4 \%$ of the patients possessed one minor criterion. No diagnosis of ARVD was made on echocardiography or EKG

\section{Conclusion}

CMR's increased sensitivity is able to detect many more abnormalities but these rarely translate into changes in clinical practice unless in the context of a diagnosis of ARVD. Although a normal CMR is reassuring and likely precludes a favorable outcome $1 / 3$ of patients still underwent further EPS testing with $1 / 2$ of those resulting in no further intervention. This details that CMR may be a more sensitive tool to exclude malignant causes of ventricular arrhythmia but this has not changed current practice patterns.

Published: 2 February 2011

doi:10.1186/1532-429X-13-S1-P250

Cite this article as: Sharma and Paterson: Role of MRI in the diagnosis and prognosis of ventricular arrhythmias. Journal of Cardiovascular Magnetic Resonance 2011 13(Suppl 1):P250.

\section{Biomed Central}

() 2011 Sharma and Paterson; licensee BioMed Central Ltd. This is an open access article distributed under the terms of the Creative Commons Attribution License (http://creativecommons.org/licenses/by/2.0), which permits unrestricted use, distribution, and reproduction in any medium, provided the original work is properly cited. 